\title{
Miocarditis fulminante en adultos por el virus de la influenza B: reporte de dos casos y revisión de la literatura
}

Edwin Silva ${ }^{1}$, Juan Sebastián Montenegro², María Camila Estupiñán ${ }^{1,3}$, Gerson Arias", Juan Pablo Osorio ${ }^{1}$, Claudia Marcela Poveda², Ricardo Buitrago ${ }^{2}$

${ }^{1}$ Grupo de Investigación en Enfermedades Infecciosas, Servicio de Infectología, Fundación Clínica Shaio, Bogotá, D.C., Colombia

${ }^{2}$ Grupo de Investigación en Enfermedades Infecciosas, Servicio de Medicina Crítica y Cuidado Intensivo, Fundación Clínica Shaio, Bogotá, D.C., Colombia

${ }^{3}$ Grupo de Medicina Interna, Universidad de la Sabana, Chía, Colombia

La miocarditis es una enfermedad inflamatoria del miocardio. Las infecciones virales son la causa más común, aunque también puede deberse a reacciones de hipersensibilidad y de etiología autoinmunitaria, entre otras.

El espectro clínico de la enfermedad es variado y comprende desde un curso asintomático, seguido de dolor torácico, arritmias y falla cardiaca aguda, hasta un cuadro fulminante. El término 'fulminante' se refiere al desarrollo de un shock cardiogénico con necesidad de soporte vasopresor e inotrópico o dispositivos de asistencia circulatoria, ya sea oxigenación por membrana extracorpórea o balón de contrapulsación intraaórtico. Cerca del $10 \%$ de los casos de falla cardiaca por miocarditis corresponde a miocarditis fulminante. La miocarditis por influenza se considera una condición infrecuente; no obstante, su incidencia ha aumentado desde el 2009 a raíz de la pandemia de influenza por el virus AH1N1. Por su parte, la miocarditis por influenza de tipo B sigue siendo una condición infrecuente. Se describen aquí dos casos confirmados de miocarditis fulminante por el virus de la influenza $B$ atendidos en un centro cardiovascular, que requirieron dispositivos de asistencia circulatoria mecánica.

Palabras clave: miocarditis; virus de la influenza B; adulto; shock cardiogénico; contrapulsador intraaórtico; oxigenación por membrana extracorpórea; circulación asistida.

Fulminant myocarditis due to the influenza B virus in adults: Report of two cases and literature review

Myocarditis is an inflammatory disease of the myocardium. Viral infections are the most common cause, although it can also be due to hypersensitivity reactions and autoimmune etiology, among other causes.

Recibido: $31 / 07 / 18$

The clinical spectrum of the disease is varied, from an asymptomatic course, followed by chest pain, arrhythmias, and acute heart failure, to a fulminant episode. The term fulminant refers to the development of cardiogenic shock with a need for vasopressor support and

Citación:

Silva E, Montenegro JS, Estupiñán MC, Arias G, Osorio JP, Poveda CM, et al. Miocarditis fulminante en adultos por el virus de la influenza B: reporte de dos casos y revisión de la literatura. Biomédica. 2019;39(Supl.2):11-19.

https://doi.org/10.7705/biomedica.v39i3.4645

Correspondencia:

Edwin Silva, Grupo de Investigación en

Enfermedades Infecciosas, Servicio de Infectología, Fundación Clínica Shaio, Diagonal 115A № 70C-75,

Bogotá, D.C., Colombia

Teléfono: (571) 6139178

edwin.silva@shaio.org

Contribución de los autores:

Edwin Silva: concepción y diseño del trabajo Juan Sebastián Montenegro y María Camila Estupiñán: recolección de datos

Todos los autores participaron en el análisis y la discusión de los datos, la revisión bibliográfica y la escritura del manuscrito.

Financiación:

El presente estudio no recibió financiación alguna. 
membrana extracorpórea (Extracorporeal Membrane Oxygenation, ECMO) o balón intraaórtico de contrapulsación, con lo que se logra la recuperación en la mayoría de los pacientes (2).

Entre los virus causantes se encuentran el virus Coxsackie, el adenovirus, el eritrovirus B19 y el virus de la influenza, entre otros. La miocarditis causada por este último se ha considerado una condición infrecuente (1); no obstante, su incidencia ha aumentado desde el 2009 a raíz de la pandemia de influenza por el virus AH1N1 (3).

En una revisión sistemática realizada por el grupo de Hékimian (3), se encontraron 184 casos reportados de miocarditis asociada al virus de la influenza, la mayoría de ellos por el subtipo AH1N1/2009. De los casos reportados, 48 progresaron a shock cardiogénico y requirieron soporte de asistencia circulatoria mecánica, en tanto que la mortalidad fue del $33 \%$. La miocarditis fulminante por el virus de la influenza $B$ es una condición poco descrita.

En la presente revisión, se analizan los casos de miocarditis por este virus en población adulta reportados en la literatura médica y se presentan dos casos de miocarditis fulminante, uno de ellos mortal.

\section{Reporte de casos}

\section{Caso 1}

Se trata de una mujer de 21 años de edad, previamente sana, que acudió al servicio de urgencias por un síncope y antecedentes de tres días de tos con expectoración hialina, malestar general y fiebre.

En el momento del ingreso estaba hipotensa y taquicárdica, no respondió a la reanimación con cristaloides y requirió soporte vasoactivo en altas dosis. El electrocardiograma evidenció taquicardia sinusal sin otros cambios, la radiografía de tórax fue normal y en el ecocardiograma se encontró disfunción ventricular grave, fracción de eyección del ventrículo izquierdo (FEVI) estimada de menos del $10 \%$, hipertrofia ventricular izquierda con hipocinesia generalizada y derrame pericárdico leve (100 ml), así como troponina I elevada (248 ng/L; valor de referencia 0,00-15,6 ng/L).

Su condición clínica se deterioró rápidamente y progresó a shock cardiogénico resistente al tratamiento y actividad eléctrica sin pulso. Durante la reanimación cardiopulmonar avanzada se le implantó un sistema ECMO venoarterial periférico.

Se tuvo sospecha de miocarditis viral como desencadenante del shock cardiogénico, por lo cual se indicó manejo antiviral empírico con oseltamivir y se solicitó un estudio de reacción en cadena de la polimerasa en tiempo real (quantitative-polymerase chain reaction, RT-PCR) múltiple para la detección de virus respiratorios en una muestra de hisopado nasofaríngeo, con la cual se confirmó la presencia del virus de la influenza B.

Pese a la colocación del dispositivo de asistencia circulatoria, la paciente presentó disfunción orgánica múltiple e isquemia crítica de miembros inferiores, que requirió canulación venoarterial central y terapia de reemplazo renal continua mediante hemofiltración. Su condición hemodinámica no mejoró con el tratamiento y después de 72 horas de ECMO venoarterial periférico y 36 horas de ECMO venoarterial central, la paciente falleció. Dada su condición hemodinámica, no se pudo hacer el estudio de resonancia magnética cardiaca. 


\section{Caso 2}

Se trata de una mujer de 62 años de edad que cinco años antes había tenido cáncer de seno tratado con cuadrantectomía, quimioterapia y radioterapia, y antecedentes relevantes de palpitaciones, por lo que se le hizo un ecocardiograma ambulatorio con resultado normal.

La paciente acudió a urgencias por cinco días de malestar, odinofagia, tos con expectoración blanquecina y disnea. En la valoración inicial estaba hipotensa y requirió soporte vasopresor. El electrocardiograma evidenció taquicardia sinusal y bajo voltaje. La troponina I presentó una importante elevación (13,373 ng/L; valor de referencia: 0,00-15,6 ng/L). El ecocardiograma reveló disfunción sistólica grave del ventrículo izquierdo con FEVI de $15 \%$, disfunción diastólica de tipo 1, disfunción sistólica grave del ventrículo derecho y derrame pericárdico anterior de 80 a $100 \mathrm{ml}$, aproximadamente, sin repercusión hemodinámica. Se hizo, además, arteriografía coronaria, la cual no evidenció lesiones.

Se consideró la posibilidad de una miocarditis fulminante con síntomas respiratorios notorios; se sospechó contagio que sugería una enfermedad similar a la influenza, por lo que se inició el manejo empírico con oseltamivir y se solicitó una RT-PCR múltiple para la detección de virus respiratorios en muestra de hisopado nasofaríngeo. La evolución no fue la adecuada y, por ello, se recurrió a la contrapulsación con balón intraaórtico.

El análisis de la muestra del hisopado nasofaríngeo mediante RT-PCR detectó el virus de la influenza $B$. Se completaron cinco días de tratamiento con oseltamivir y el balón de contrapulsación se retiró al cuarto día ante la rápida mejoría de la condición clínica de la paciente. El ecocardiograma de control mostró una FEVI calculada de 30 \% y la resonancia cardiaca correspondió con el diagnóstico de miocarditis (figura 1). La paciente egresó a los pocos días.

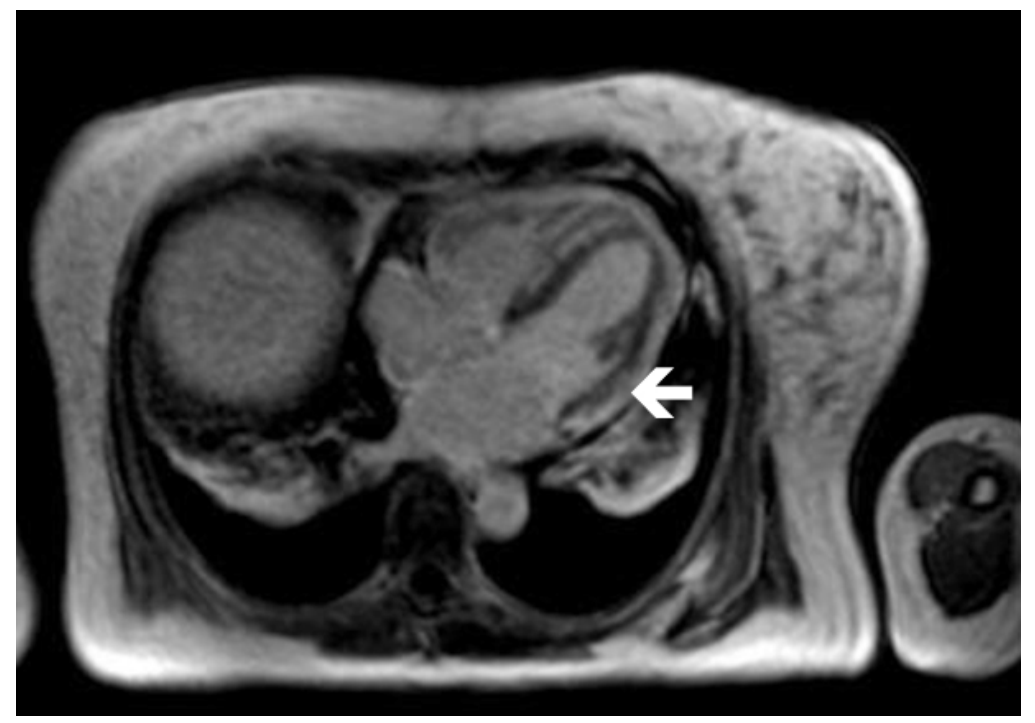

Figura 1. Resonancia magnética cardiaca. Secuencia con contraste tardío, la flecha señala la retención subepicárdica global de gadolinio, lo que indica la presencia de fibrosis del miocardio ventricular y es sugestivo de miocarditis.

Fuente: Departamento de Imágenes Diagnósticas, Fundación Clínica Shaio, Bogotá, D.C., Colombia 


\section{Revisión de la literatura}

La revisión de la literatura en lengua inglesa se hizo en PubMed Medline con los términos MeSH Influenza $B$ virus y Myocarditis en el periodo comprendido entre el $1^{\circ}$ de enero de 1946 y el 31 de diciembre de 2018; asimismo, se revisaron las referencias de las publicaciones encontradas para buscar casos adicionales. Se excluyeron los casos con información insuficiente y aquellos en población pediátrica, dado que la experiencia de los autores se circunscribe a la atención de población adulta (>15 años).

Se encontraron nueve casos reportados de miocarditis por el virus de la influenza B en población adulta (3-8), de los cuales se descartaron seis casos en población pediátrica (9-14); otras tres publicaciones se descartaron por información insuficiente (15-17).

Los pacientes de los informes seleccionados tenían un rango de edad de 15 a 81 años, con una media de 39,7 años, eran mayoritariamente del sexo femenino $(66,6 \%)$ y el tiempo de evolución de los síntomas antes de la consulta había sido de 4,7 días en promedio (cuadro 1).

Solo en el caso de un hombre se presentó síndrome de dificultad respiratoria del adulto (SDRA). Tres pacientes no requirieron soporte con dispositivos de asistencia circulatoria y en los reportes no se mencionó la necesidad de soporte vasopresor; en los restantes seis casos se había presentado shock cardiogénico (miocarditis fulminante) y se requirió algún tipo de asistencia circulatoria (ECMO en cinco pacientes).

Cuadro 1. Casos de miocarditis por el virus de la influenza B en población adulta reportados en la literatura médica

\begin{tabular}{|c|c|c|c|c|c|c|c|c|c|c|c|c|}
\hline Autor & Año & $\begin{array}{l}\text { Edad } \\
\text { (años) }\end{array}$ & Sexo & $\begin{array}{l}\text { Días de } \\
\text { síntomas } \\
\text { antes } \\
\text { de la } \\
\text { consulta }\end{array}$ & $\begin{array}{l}\text { Método } \\
\text { diagnóstico }\end{array}$ & $\begin{array}{l}\text { Presentación } \\
\text { clínica }\end{array}$ & $\begin{array}{l}\text { Troponina } \\
\text { (ng/L) }\end{array}$ & $\begin{array}{l}\text { Radiografía } \\
\text { de tórax }\end{array}$ & $\begin{array}{l}\text { FEVI } \\
(\%)\end{array}$ & $\begin{array}{l}\text { Soporte } \\
\text { circulatorio } \\
\text { mecánico } \\
\text { (días) }\end{array}$ & $\begin{array}{l}\text { Tratamiento } \\
\text { (días) }\end{array}$ & Supervivencia \\
\hline $\begin{array}{l}\text { Muneuchi, } \\
\text { et al. (5) }\end{array}$ & 2009 & 15 & $M$ & 2 & $\begin{array}{l}\text { Prueba } \\
\text { rápida de } \\
\text { antígenos }\end{array}$ & Imitando SCA & 1.530 & NR & 63 & No & $\begin{array}{l}\text { Zanamivir } \\
\text { (NR) }\end{array}$ & Sí \\
\hline $\begin{array}{l}\text { Taremi, } \\
\text { et al. (6) }\end{array}$ & 2013 & 52 & $\mathrm{~F}$ & 5 & $\begin{array}{l}\text { Aspirado } \\
\text { traqueal } \\
\text { PCR }\end{array}$ & $\begin{array}{l}\text { Shock } \\
\text { cardiaco }\end{array}$ & 12.600 & $\begin{array}{l}\text { Opacidades } \\
\text { difusas } \\
\text { bilaterales }\end{array}$ & 10 & ECMO (7) & $\begin{array}{l}\text { Oseltamivir } \\
(7)\end{array}$ & Sí \\
\hline $\begin{array}{l}\text { Geladari, } \\
\text { et al. (7) }\end{array}$ & 2016 & 81 & $\mathrm{~F}$ & 2 & $\begin{array}{l}\lg M \\
\text { Influenza B }\end{array}$ & $\begin{array}{l}\text { Miocarditis } \\
\text { aguda }\end{array}$ & 2.487 & $\begin{array}{l}\text { Opacidades } \\
\text { difusas } \\
\text { bilaterales }\end{array}$ & 60 & No & $\begin{array}{l}\text { Oseltamivir } \\
\text { (NR) }\end{array}$ & Sí \\
\hline $\begin{array}{l}\text { Chang, } \\
\text { et al. (4) }\end{array}$ & 2016 & 43 & $M$ & 4 & $\begin{array}{l}\text { Aspirado } \\
\text { traqueal } \\
\text { PCR }\end{array}$ & $\begin{array}{l}\text { Miocarditis } \\
\text { aguda y SDRA }\end{array}$ & 60 & SDRA & 60 & No & $\begin{array}{l}\text { Oseltamiivir } \\
\text { (5) }\end{array}$ & Sí \\
\hline $\begin{array}{l}\text { Siskin, } \\
\text { et al. (8) }\end{array}$ & 2017 & 22 & $\mathrm{~F}$ & 14 & $\begin{array}{l}\text { Hisopado } \\
\text { nasofaríngeo } \\
\text { PCR }\end{array}$ & $\begin{array}{l}\text { Shock } \\
\text { cardiaco }\end{array}$ & 13.000 & NR & 10 & Impella (8) & $\begin{array}{l}\text { Oseltamivir } \\
\text { (NR) }\end{array}$ & Sí \\
\hline $\begin{array}{l}\text { Hékimian, } \\
\text { et al. (3) }\end{array}$ & 2018 & 28 & $\mathrm{~F}$ & 3 & $\begin{array}{l}\text { Muestra } \\
\text { respiratoria } \\
\text { PCR }\end{array}$ & $\begin{array}{l}\text { Shock } \\
\text { cardiaco }\end{array}$ & 3.920 & Normal & 30 & ECMO (5) & $\begin{array}{l}\text { Oseltamivir } \\
\text { (5) }\end{array}$ & Sí \\
\hline $\begin{array}{l}\text { Hékimian, } \\
\text { et al. (3) }\end{array}$ & 2018 & 35 & $F$ & 3 & LBA - PCR & $\begin{array}{l}\text { Shock } \\
\text { cardiaco }\end{array}$ & 3.460 & Normal & 25 & ECMO (5) & $\begin{array}{l}\text { Oseltamivir } \\
\text { (5) }\end{array}$ & Sí \\
\hline $\begin{array}{l}\text { Hékimian, } \\
\text { et al. (3) }\end{array}$ & 2018 & 43 & $\mathrm{~F}$ & 5 & LBA - PCR & $\begin{array}{l}\text { Shock } \\
\text { cardiaco }\end{array}$ & 6.940 & Normal & 30 & ECMO (7) & $\begin{array}{l}\text { Oseltamivir } \\
(10)\end{array}$ & Sí \\
\hline $\begin{array}{l}\text { Hékimian, } \\
\text { et al. (3) }\end{array}$ & 2018 & 39 & $M$ & 5 & $\begin{array}{l}\text { Muestra } \\
\text { respiratoria } \\
\text { PCR }\end{array}$ & $\begin{array}{l}\text { Shock } \\
\text { cardiaco }\end{array}$ & 6.973 & Normal & 20 & ECMO (9) & $\begin{array}{l}\text { Oseltamivir } \\
(10)\end{array}$ & Sí \\
\hline
\end{tabular}

PCR: reacción en cadena de la polimerasa; IgM: inmunoglobulina M; LBA: lavado broncoalveolar; SCA: síndrome coronario agudo; SDRA: síndrome agudo de dificultad

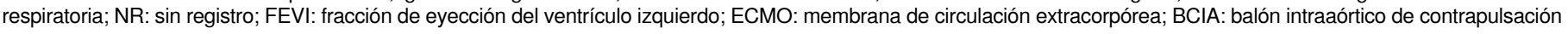


A todos los pacientes de los casos reportados se les suministró tratamiento antiviral con inhibidores de la neuraminidasa, la mayoría con oseltamivir (solo un caso con zanamivir), durante cinco días. No se presentó ningún caso fatal en los informes revisados.

\section{Discusión}

El virus de la influenza es una de las principales causas de infección respiratoria aguda grave en el adulto, y afecta anualmente entre el 5 y el $15 \%$ de la población mundial (18).

La miocarditis es una complicación poco frecuente de la influenza, aunque con un incremento en los últimos años a partir de la pandemia por el virus de la influenza AH1N1/2009 (2), cuya verdadera incidencia puede estar siendo subestimada (19). La gran mayoría de los casos de miocarditis por influenza se relacionan con el virus de tipo A y, en menor proporción, con el de tipo B. En la serie de Hékimiam, et al. (3), solo el $8 \%$ de los casos de miocarditis por influenza fueron secundarios a la infección por el virus de la influenza B. El virus de la influenza AH1N1 presenta tropismo por el músculo estriado y el número de reportes de casos de rabdomiólisis con falla renal aguda es significativo (20). La fisiopatología de este proceso es aún tema de debate, pero como mecanismo causal se ha propuesto la infiltración del virus en el músculo estriado (tanto esquelético, como cardiaco), así como la circulación de toxinas víricas causantes del daño muscular (21).

Por su parte, la influenza B se describe generalmente como una enfermedad leve y sus complicaciones fatales son raras (22).

En la presente revisión se analizaron nueve casos de miocarditis por el virus de la influenza $B$ reportados en la literatura, de los cuales seis cumplían con la definición de miocarditis fulminante. Los mecanismos fisiopatológicos de la miocarditis fulminante no se han dilucidado del todo (23), pero se han planteado diferentes teorías para explicar el daño miocárdico que produce, por ejemplo, la que plantea que la lesión directa del cardiomiocito es mediada por proteasas virales que inducen citólisis extensa y temprana, lo que lleva finalmente al desarrollo de la insuficiencia cardiaca (24); este proceso también expone proteínas intracelulares que las células presentadoras de antígenos reconocen como epítopos y que, así, inducen la activación de linfocitos T CD4 y su diferenciación a Th1 y Th17, lo cual favorecería el proceso autoinflamatorio mediante la activación y el reclutamiento de macrófagos, neutrófilos, linfocitos T CD8 y células B autorreactivas, células estas que liberan autoanticuerpos contra la célula cardiaca, lo que perpetúa el daño miocárdico (25).

Sin embargo, el curso hiperagudo puede explicarse mejor por la hipótesis de la 'tormenta de citocinas', pues se ha postulado que la liberación de las interleucinas 1 y 6 y del factor de necrosis tumoral, podría explicar el carácter fulminante de esta enfermedad (24).

En la presente serie de casos de miocarditis por el virus de la influenza B, el tiempo de evolución de los síntomas antes de la consulta fue apenas de 4,7 días; de hecho, si se excluye un caso con 14 días de evolución previa, el tiempo promedio para los ocho casos restantes fue de apenas 3,6 días. Este hallazgo evidencia cuán aguda es la evolución de esta condición, el curso frecuente hacia una miocarditis fulminante y la importancia del inicio temprano del tratamiento antiviral y del soporte circulatorio en caso de requerirse. 
En la revisión de Ukimura, et al., sobre miocarditis por influenza AH1N1 en Japón (26), el $28 \%$ de los casos fue mortal; un dato similar se reportó en la revisión sistemática de Hékimiam, et al. (3), con una mortalidad de $31 \%$ para los 173 casos de miocarditis por el virus de la influenza $A$ de la serie. En esa serie no hubo casos fatales, lo cual, paradójicamente, podría indicar un comportamiento más benigno del virus de tipo $\mathrm{B}$, a pesar de la mayor incidencia de miocarditis fulminante.

En el presente reporte, se presenta el primer caso de mortalidad por miocarditis fulminante por el virus de la influenza B en población adulta, el cual afectó a una paciente joven y sin antecedentes importantes de enfermedad, quien presentó un cuadro clínico agudo con deterioro hemodinámico grave que desembocó en actividad eléctrica sin pulso a las pocas horas de haber ingresado al hospital, por lo que requirió una reanimación de 120 minutos, aproximadamente, hasta el inicio del soporte circulatorio con ECMO venoarterial, lo cual representaba un pronóstico adverso para la supervivencia, a pesar del manejo médico oportuno.

La presunción diagnóstica de la miocarditis fulminante exige un alto nivel de sospecha y, usualmente, recursos diagnósticos que no siempre están disponibles; no obstante, la presencia de shock cardiogénico en adultos jóvenes, usualmente precedida de un cuadro viral, es suficiente para establecer un índice adecuado de sospecha. Los hallazgos de laboratorio son inespecíficos, los biomarcadores cardiacos generalmente están elevados y se pueden encontrar alteraciones variables en el electrocardiograma $(27,28)$. El ecocardiograma y la resonancia magnética permiten demostrar el compromiso de la función cardiaca y evidenciar áreas de inflamación, necrosis y fibrosis $(29,30)$. El estudio histopatológico es el método diagnóstico estándar; sin embargo, su disponibilidad y su carácter invasivo en pacientes que habitualmente presentan inestabilidad hemodinámica hacen que este recurso sea de aplicación limitada (31).

El diagnóstico de las infecciones virales respiratorias incluye el uso de pruebas rápidas de antígenos, pruebas de anticuerpos por fluorescencia directa y cultivos virales cuya eficacia diagnóstica es variable (sensibilidad de 44 a $95 \%$ y especificidad de 74 a $100 \%$ ). Además, la disponibilidad de estas técnicas depende de los recursos, de personal especializado para su interpretación y de la tecnología con la que cuente cada institución (32).

Estas pruebas han sido reemplazadas por el diagnóstico molecular mediante PCR, con diferentes plataformas disponibles en el mercado. En los casos que se presentan aquí, se usó el panel respiratorio FilmArray ${ }^{\mathrm{TM}}$ (bioMérieux, BioFire, Salt Lake City, UT, USA), el cual combina de forma automatizada la preparación y extracción de ácidos nucleicos, la PCR múltiple anidada y la detección de los blancos amplificados, e identifica 20 virus y bacterias, incluidos los de influenza $A(H 1 N 1, H 3 N 2$ y H1N1pandémica-2009) e influenza $B$ (33). El rendimiento diagnóstico del panel respiratorio FilmArray ${ }^{\mathrm{TM}}$ para la identificación del virus de la influenza, ha sido evaluado en diferentes estudios con resultados de sensibilidad y especificidad para el virus de la influenza $A$ de 86,2 a $100 \%$ y del $100 \%$, respectivamente, y para el de la influenza B, de 77,3 a $100 \%$ y $100 \%$, respectivamente $(34,35)$. Dado que la sensibilidad y la especificidad de las técnicas de diagnóstico molecular por PCR son mejores que los métodos convencionales, actualmente constituyen la mejor herramienta de diagnóstico etiológico para las infecciones virales respiratorias. 
En los casos de miocarditis por el virus de la influenza $B$ reportados en esta revisión, la mayoría correspondió a mujeres $(66,6 \%$ ) que no tenían compromiso respiratorio concomitante $(88,8 \%)$, en tanto que el único caso con SDRA se presentó en un hombre (4). De los seis casos de miocarditis fulminante, cinco fueron en mujeres. Esta presentación diversa de la miocarditis fulminante por el virus de la influenza B (aparentemente con mejor pronóstico de supervivencia), sugiere claramente la presencia de una enfermedad diferenciada.

\section{Conclusiones}

Se reportan los casos de dos mujeres con miocarditis fulminante por el virus de la influenza $B$, una de las cuales falleció. Esta miocarditis es más frecuente en mujeres jóvenes sin compromiso respiratorio concomitante, generalmente con un cuadro agudo que progresa a shock cardiogénico (miocarditis fulminante) y que, en la mayoría de los casos, sobreviven. El tratamiento consiste en el inicio temprano del uso de antivirales y de dispositivos de asistencia circulatoria cuando se requiere. El comportamiento clínico de la miocarditis por el virus de la influenza B sugiere un mecanismo fisiopatológico diferente al de la miocarditis por el virus de la influenza A.

El presente reporte se ajustó a la Declaración de Helsinki y se garantizó la confidencialidad de la historia clínica de los pacientes. La información suministrada es crucial para mejorar la atención de otros pacientes de acuerdo a las buenas prácticas clínicas en investigación.

\section{Agradecimientos}

Al Grupo de Laboratorio Clínico de la Fundación Clínica Shaio, por el procesamiento de las muestras en la RT-PCR múltiple para virus respiratorios.

\section{Referencias}

1. Estabragh ZR, Mamas MA. The cardiovascular manifestations of influenza: A systematic review. Int J Cardiol. 2013;167:2397-403. https://doi.org/10.1016/j.ijcard.2013.01.274

2. Ukimura A, Izumi T, Matsumori A, Clinical Research Committee on Myocarditis Associated with 2009 Influenza A (H1N1) Pandemic in Japan organized by Japanese Circulation Society. A national survey on myocarditis associated with the 2009 influenza A (H1N1) pandemic in Japan. Circ J. 2010;74:2193-9. https://doi.org/10.1253/circj.CJ-10-0452

3. Hékimiam G, Jovanovic T, Bréchot N, Lebreton G, Leprince P, Trouillet JL, et al. When the heart gets the flu: Fulminant influenza B myocarditis: A case-series report and review of the literature. J Crit Care. 2018;47:61-4. https//doi.org/10.1016/j.jcrc.2018.06.001

4. Chang HL, Hsu JF, Tsai YM, Lin SY, Kuo HF, Yang CJ. Acute respiratory distress syndrome and acute myocarditis developed in a previously healthy adult with influenza B. BMC Pulm Med. 2016;16:1. https//doi.org/10.1186/s12890-015-0163-3

5. Muneuchi J, Kanaya Y, Takimoto T, Hoshina T, Kusuhara K, Hara T. Myocarditis mimicking acute coronary syndrome following influenza B virus infection: A case report. Cases J. 2009;2:6809. https//doi.org/10.4076/1757-1626-2-6809

6. Taremi M, Amoroso A, Nace HL, Gilliam BL. Influenza B-induced refractory cardiogenic shock: A case report. BMC Infect Dis. 2013;13:452. https//doi.org/10.1186/1471-2334-13-452

7. Geladari EV, Vallianou NG, Kratimenos Th, Trigkidis K, Kokkinakis E. Myocarditis due to influenza B virus: The importance of early antiviral therapy. J Cardiol Clin Res. 2016;4:1079.

8. Siskin M, Rao S, Rapkiewicz A, Bangalore S, Garshick M. A case of cardiogenic shock secondary to complement-mediated myopericarditis from influenza B infection. Can J Cardiol. 2017;33:1335. https//doi.org/10.1016/j.cjca.2017.06.006

9. Frank H, Wittekind C, Liebert UG, Siekmeyer M, Siekmeyer W, Schuster V, et al. Lethal influenza B myocarditis in a child and review of the literature for pediatric age groups. Infection. 2010;38:231-5. https//doi.org/10.1007/s15010-010-0013-4 
10. Dalton HJ. Fighting the flu: The rise of the machine. Pediatr Crit Care Med. 2004;5:415-7. https//doi.org/10.1097/01.PCC.0000128886.08534.9E

11. Tabbutt S, Leonard M, Godinez RI, Sebert M, Cullen J, Spray TL, et al. Severe influenza B myocarditis and myositis. Pediatr Crit Care Med. 2004;5:403-6. https//doi.org/10.1097/01.PCC.0000123555.10869.09

12. Akhtar N, Ni J, Stromberg D, Rosenthal GL, Bowles NE, Towbin JA. Tracheal aspirate as a substrate for polymerase chain reaction detection of viral genome in childhood pneumonia and myocarditis. Circulation. 1999;99:2011-8.

13. Shetty AK, Desselle B. A child with chest pain. Clin Pediatr (Phila). 1998;37:317-9. https://doi.org/10.1177/000992289803700507

14. Craver RD, Sorrells K, Gohd R. Myocarditis with influenza B infection. Pediatr Infect Dis J. 1997;16:629-30.

15. Yoshimizu N, Tominaga T, Ito T, Nishida Y, Wada Y, Sohmiya K, et al. Repetitive fulminant influenza myocarditis requiring the use of circulatory assist devices. Intern Med. 2014;53:109-14. https://doi.org/10.2169/internalmedicine.53.1117

16. McGregor D, Henderson S. Myocarditis, rhabdomyolysis and myoglobinuric renal failure complicating influenza in a young adult. N Z Med J. 1997;110:237.

17. Ray CG, Icenogle TB, Minnich LL, Copeland JG, Grogan TM. The use of intravenous ribavirin to treat influenza virus-associated acute myocarditis. J Infect Dis. 1989;159:829-36. Erratum in: J Infect Dis. 1989;160:564.

18. Mertz D, Kim TH, Johnstone J, Lam PP, Science M, Kuster SP, et al. Populations at risk for severe or complicated influenza illness: Systematic review and meta-analysis. BMJ. 2013;347:f5061. https://doi.org/10.1136/bmj.f5061

19. Datta R, Helou E, Tucker M, Blessy J, Martinello R, Malinis M. Detection of influenza myocarditis using national healthcare safety network surveillance definitions accounting for fever in older adults. Infect Control Hosp Epidemiol. 2018;39:1145-7. https://doi.org/10.1017/ ice.2018.147

20. Lai CC, Wang CY, Lin HI. Rhabdomyolysis and acute kidney injury associated with 2009 pandemic influenza A(H1N1). Am J Kidney Dis. 2010;55:615. https://doi.org/10.1053/j.ajkd.2010.01.002

21. Ayala E, Kagawa FT, Wehner JH, Tam J, Upadhyay D. Rhabdomyolysis associated with 2009 influenza A(H1N1). JAMA. 2009;302:1863-4. https://doi.org/10.1001/jama.2009.1582

22. Rezkalla SH, Kloner RA. Influenza-related viral myocarditis. WMJ. 2010;109:209-13.

23. Ginsberg F, Parrillo JE. Fulminant myocarditis. Crit Care Clin. 2013;29:465-83. https://doi.org/10.1016/j.ccc.2013.03.004

24. Liu PP, Mason JW. Advances in the understanding of myocarditis. Circulation. 2001;104:1076-82.

25. Reddy J, Massilamany C, Buskiewicz I, Huber SA. Autoimmunity in viral myocarditis. Curr Opin Rheumatol. 2013;25:502-8. https://doi.org/10.1097/BOR.0b013e3283620036

26. Ukimura A, Ooi Y, Kanzaki Y, Inomata T, Izumi T. A national survey on myocarditis associated with influenza $\mathrm{H} 1 \mathrm{~N} 1 \mathrm{pdm} 2009$ in the pandemic and postpandemic season in Japan. J Infect Chemother. 2013;19:426-31. https://doi.org/10.1007/s10156-012-0499-z

27. Cooper LT. Myocarditis. N Engl J Med. 2009;360:1526-38. https://doi.org/10.1056/NEJMra0800028

28. Domínguez F, Kühl U, Pieske B, García P, Tschöpe C. Actualización sobre miocarditis y miocardiopatía inflamatoria: el resurgir de la biopsia endomiocárdica. Rev Esp Cardiol. 2016; 69:178-87. https://doi.org/10.1016/j.recesp.2015.10.018

29. Mavrogeni S, Manoussakis MN. Myocarditis as a complication of influenza A (H1N1): Evaluation using cardiovascular magnetic resonance imaging. Hellenic $\mathrm{J}$ Cardiol. 2010;51:379-80

30. Felker GM, Boehmer JP, Hruban RH, Hutchins GM, Kasper EK, Baughman KL, et al. Echocardiographic findings in fulminant and acute myocarditis. J Am Coll Cardiol. 2000;36:227-32. https://doi.org/10.1016/S0735-1097(00)00690-2

31. Leone O, Veinot JP, Angelini A, Baandrup UT, Basso C, Berry G, et al. 2011 consensus statement on endomyocardial biopsy from the Association for European Cardiovascular Pathology and the Society for Cardiovascular Pathology. Cardiovasc Pathol. 2012;21:24574. https://doi.org/10.1016/j.carpath.2011.10.001 
32. Ginocchio CC. Detection of respiratory viruses using non-molecular based methods. J Clin Virol. 2007;40(Suppl.1):S11-4. https://doi.org/10.1016/S1386-6532(07)70004-5

33. Babady NE. The FilmArray® respiratory panel: An automated, broadly multiplexed molecular test for the rapid and accurate detection of respiratory pathogens. Expert Rev Mol Diagn. 2013;13:779-88. https://doi.org/10.1586/14737159.2013.848794

34. Rand $\mathrm{KH}$, Rampersaud H, Houck HJ. Comparison of two multiplex methods for detection of respiratory viruses: FilmArray RP and xTAG RVP. J Clin Microbiol. 2011;49:2449-53. https://doi.org/10.1128/JCM.02582-10

35. Popowitch EB, O'Neill SS, Miller MB. Comparison of the Biofire FilmArray RP, Genmark eSensor RVP, Luminex xTAG RVPv1, and Luminex xTAG RVP fast multiplex assays for detection of respiratory viruses. J Clin Microbiol. 2013;51:1528-33. https://doi.org/10.1128/JCM.03368-12 